\title{
RELAÇÃO POROSIDADE/CIMENTO COMO PARÂMETRO DE CONTROLE NA ESTABILIZAÇÃO DE UM SOLO SILTOSO
}

\author{
POROSITY/CEMENT RATIO AS A CONTROL PARAMETER OF A \\ STABILIZED SILTY SOIL
}

\author{
Jair de Jesús Arrieta Baldovino, Ronaldo Luis dos Santos Izzo \\ Universidade Tecnológica Federal do Paraná, UTFPR, Curitiba,PR-Brasil \\ E-mail: yaderbal@hotmail.com; izzo@utfpr.edu.br
}

\begin{abstract}
RESUMO - O presente trabalho apresenta o desenvolvimento da resistência à compressão simples $\left(q_{u}\right)$ e da resistência à tração indireta $\left(q_{t}\right)$ de um solo siltoso estabilizado quimicamente. Para isso, foram moldados corpos de prova com volume de $196,35 \mathrm{~cm}^{3}$ empregando teores de cimento de $3 \%, 5 \%, 7 \%$ e $9 \%$ e posteriormente ensaiados depois de 7 dias de cura. Foi utilizada como principal parâmetro de análise dos resultados a relação porosidade/teor volumétrico de cimento $\left(\eta / C_{i v}\right)$. Os resultados exibem que com a diminuição da relação $\eta / C_{i v}$ os valores de $q_{u}$ e $q_{t}$ crescem. Assim, a diminuição da porosidade significou um aumento em $q_{u}$ e $q_{t}$. Uma equação geral de estimativa para $q_{u} e$ $q_{t}$ foi desenvolvida para estimar a resistência das misturas com o uso de $\eta / C_{i v}$ ajustada a um escalar igual a 0,45 . As equações demonstraram que é possível estimar o valor de $\mathrm{q}_{\mathrm{u}}$ e $\mathrm{q}_{\mathrm{t}}$ do solo tratado dentre as quantidades de cimento, relação $\eta / C_{i v}$ e tempo de cura empregado no presente trabalho. Finalmente, foi calculada uma relação constante $d_{\mathrm{t}} \mathrm{q}_{\mathrm{t}} / \mathrm{q}_{\mathrm{u}}=0,15$, a qual é independente dos vazios e do teor volumétrico de cimento.
\end{abstract}

Palavras-chave: Estabilização de solos; porosidade-cimento; resistência mecânica.

ABSTRACT - This paper presents the development of the unconfined compressive $\left(q_{u}\right)$ and the splitting tensile strength $\left(q_{t}\right)$ of a chemically stabilized silt soil. For this, specimens with a volume of $196,35 \mathrm{~cm}^{3}$ were prepared using cement contents of $3 \%, 5 \%, 7 \%$ and $9 \%$ and then tested after 7 days of cure. The porosity/volumetric cement content $\left(\eta / C_{\text {iv }}\right)$ was used as the main parameter of the results analysis. The results show that with the decrease in the ratio $\eta / c_{i v}$ the values of $q_{u}$ and $q_{t}$ increase. Thus, the decrease in porosity meant an increase in $q_{u}$ and $q_{t}$. A general estimation equation for $q u$ and $q t$ was developed to estimate the resistance of the mixtures with the use of $\eta / C_{i v}$ adjusted to a scalar equal to 0,45 . The equations showed that it is possible to estimate the value of qu and qt of treated soil among the amounts of cement,

Recebido em: 25/02/2019 Revisado em: 30/04/2019 Aprovado em: 03/05/2019 $\eta / C_{i v}$ ratio and curing time employed in the present work. Finally, a constant ratio of $\mathrm{q}_{\mathrm{t}} / \mathrm{q}_{\mathrm{u}}=0,15$, which is independent of the voids and the volumetric content of cement, was calculated.

Keywords: Soil stabilization; porosity-cement, strength. 


\section{INTRODUÇÃO}

Uma das metodologias para melhorar as propriedades físico-mecânicas dos solos é a adição de cimento. A metodologia tem sido utilizada para estabilizar solos para uso em camadas de pavimentos, para reforço de solos usados como suporte de fundações superficiais, proteção de encostas e taludes e para construção de fundações profundas. A metodologia vem sendo empregada há 100 anos (FIROOZI et al., 2017).

O solo-cimento é definido como uma mistura de solo e quantidades medidas de cimento Portland e água e compactado para a densidade desejada. O cimento é mais comumente empregado para aumentar a resistência de solos com granulometria mais arenosa. Quando a água é adicionada na mistura solo-cimento e logo compactada, se dá a hidratação, o que significa que são formados compostos cimentantes de hidrato de silicato de cálcio e hidrato de aluminato de cálcio e o excesso de hidróxido de cálcio [CaOH] é liberado (RONOH et al., 2014).

Lade and Trads (2014) reportaram o papel da cimentação no comportamento de solos cimentados artificialmente baseados em estudos experimentais mediante modelos elastoplásticos para estabelecer a influência do poder da cimentação no desenvolvimento da resistência do material solo-cimento. Por outra parte, Horpibulsuk et al. (2010) analisaram o desenvolvimento da resistência de misturas de uma argila siltosa misturada com cimento baseados em considerações microestruturais, estudando a influência do teor de umidade, do tempo de cura e da quantidade de cimento. A adição do cimento melhora a estrutura do solo aumentando a ligação inter-cluster e reduzindo assim o espaço dos poros. Para Horpibulsuk et al. (2010) a água influencia tanto nos produtos de hidratação como nos tamanhos dos poros, sendo a água ótima 0,8 vezes o valor da umidade ótima de compactação.

Pakbaz and Alipour (2012) pesquisaram a influência da adição de cimento Portland nas propriedades geotécnicas em um solo argiloso, com adições de cimento de 4, 6, 8 e 10\% em referência à massa seca do solo, usando três umidades de mistura, 30, 48 e $70 \%$ e empregando 7, 14 e 28 dias de cura. Os pesquisadores reportaram a resistência à compressão simples de $250 \mathrm{kPa}$ para $4 \%$ de cimento chegando a $2900 \mathrm{kPa}$ para adição de $10 \%$ de cimento, ambos aos 28 dias de cura. A massa real dos grãos (Gs) após a cura aumentou ligeiramente de 2,68 (para a amostra não estabilizada) para 2,73-2,76 (para amostras estabilizadas com cimento). Os valores do Gs das amostras tratadas diminuíram com um aumento do teor de cimento e tempo de cura.

Recentemente, Jin et al. (2018) reportaram valores de até $5000 \mathrm{kPa}$ com misturas de solo estabilizado com 3 e $15 \%$ de cimento em referência à massa seca do solo e com significativa redução da plasticidade da mistura. Consoli et al. (2013) estudaram a influência dos tipos de cimento Portland (I, III e IV) em um solo arenoso adicionando de $3 \%$ a $9 \%$ de cimento ao solo com tempos de cura de 2,7 e 28 dias. Os autores mostraram que há acréscimo de resistência $q_{u}$ de até 1600 $\mathrm{kPa}, 2600 \mathrm{kPa}$ e $1600 \mathrm{kPa}$ para o cimento I, III e IV, respectivamente. Outros autores como Por et al. (2017) apresentaram resultados de compressão simples com adição de 5 e 10\%, em relação à massa seca do solo, obtendo resultados de até $1500 \mathrm{kPa}$ aos 14 dias e $2900 \mathrm{kPa}$ aos 28 dias de cura, esses resultados são semelhantes aos reportados por Consoli et al. (2013). Os autores também reportam a diminuição significativa do índice de plasticidade e expansão do solo, diminuindo de $8 \%$ (sem adição de cimento) para $0 \%$ com adição de cimento.

Como foi visto anteriormente, o tópico de estabilização de solos com cimento vem sendo bastante pesquisado, sobretudo em lugares onde os solos por suas próprias características físico mecânicas não podem ser empregados na engenharia geotécnica nem na construção civil. Assim, o presente trabalho presenta os efeitos da adição de cimento CP V-ARI na resistência à 
compressão simples e tração por compressão diametral de um solo siltoso da região metropolitana de Curitiba/PR. O artigo também apresenta os fatores que influenciam no acréscimo ou diminuição da resistência mecânica como o teor de cimento, a porosidade e o teor volumétrico de cimento.

\section{PROGRAMA EXPERIMENTAL}

O programa experimental foi dividido em duas etapas: a primeira foi a realização dos ensaios de caracterização do solo e do cimento: granulometria do solo de acordo à norma americana ASTM D2487 (ASTM 2011), limites de Atterberg do solo de acordo às normas brasileiras NBR 7180 (ABNT 2016) e NBR 6459 (ABNT 2016), a massa especifica real dos grãos do solo de acordo à norma ASTM D854 (ASTM 2014), massa especifica real dos grãos do cimento de acordo à norma brasileira NBR 16605 (ABTN 2017) e as propriedades de compactação do solo e das misturas solo-cimento nas três energias (normal, intermediária e modificada) de acordo à norma brasileira NBR 7182 (ABNT 2016); e a segunda etapa, consistiu-se na moldagem, cura e rompimento dos corpos de prova solo-cimento submetidos a ensaios de compressão simples e tração por compressão diametral.

\subsection{Materiais}

No presente trabalho foram utilizados três materiais: solo, cimento Portland CP V e água destilada. A amostra de solo foi coletada na zona oeste da cidade de Curitiba (Brasil) no município de São José dos Pinhais de maneira manual em estado deformado, evitando uma possível contaminação e em quantidade suficiente para a realização de todos os ensaios. O solo foi coletado em um talude rodoviário a $2,5 \mathrm{~m}$ de profundidade. Segundo o Sistema Unificado de Classificação de Solos, o solo é classificado como um silte elástico arenoso (MH). Quanto a caracterização física do solo, o material apresentou uma pequena variação nos percentuais de cada tipo de componente, na qual a porcentagem de areia média alcançou $12 \%$; enquanto a areia fina chegou em $18 \%$; para o silte se obteve $60 \%$ e completando a fração fina, o percentual de argila foi de $5 \%$, sendo a porcentagem de silte, compreendia na fração de $(0,002 \mathrm{~mm}<$ diâmetro $<0,075$ $\mathrm{mm}$ ) compõe a maior parcela do solo. Os resultados dos ensaios de caracterização física do solo são apresentados na Tabela 1. O cimento foi fornecido por um fabricante local. A Tabela 2 apresenta as propriedades físico-químicas do cimento. As propriedades químicas foram fornecidas pelo fabricante $\mathrm{e}$ as físicas foram calculadas no laboratório. De acordo à Tabela 2 o cimento $\mathrm{CP} V$ tem uma massa específica de 3,11. 
Tabela 1. Propriedades físicas do solo

\begin{tabular}{|l|c|}
\hline \multicolumn{1}{|c|}{ Propriedades } & Valor \\
\hline Limite de liquides, $\%$ & 50,82 \\
\hline Limite de plasticidade, $\%$ & 35,96 \\
\hline Índice de plasticidade, $\%$ & 14,86 \\
\hline Densidade real dos grãos & 2,62 \\
\hline Areia grossa $(0,6 \mathrm{~mm}<$ diâmetro $<2 \mathrm{~mm}), \%$ & 5 \\
\hline Areia média $(0,2 \mathrm{~mm}<$ diâmetro $<0,6 \mathrm{~mm}), \%$ & 12 \\
\hline Areia fina $(0,06 \mathrm{~mm}<$ diâmetro $<0,2 \mathrm{~mm}), \%$ & 18 \\
\hline Silte $(0,002 \mathrm{~mm}<$ diâmetro $<0,06 \mathrm{~mm}), \%$ & 60 \\
\hline Argila (diâmetro $<0,002 \mathrm{~mm}), \%$ & 5 \\
\hline Diâmetro efetivo $\left(\mathrm{D}_{10}\right), \mathrm{mm}$ & 0,003 \\
\hline Diâmetro médio $\left(\mathrm{D}_{50}\right), \mathrm{mm}$ & 0,038 \\
\hline Coeficiente de uniformidade $\left(\mathrm{C}_{\mathrm{u}}\right)$ & 12,67 \\
\hline Coeficiente de curvatura $\left(\mathrm{C}_{\mathrm{c}}\right)$ & 0,88 \\
\hline Classificação $(\mathrm{SUCS})$ & $\mathrm{MH}$ \\
\hline Cor & Amarelo \\
\hline
\end{tabular}

Para a realização de todos os ensaios de caracterização do solo, das misturas solocimento e para a moldagem de corpos de prova foi usada água destilada a $24 \pm 3^{\circ} \mathrm{C}$ para evitar reações não desejadas e limitar o número de variáveis no estudo.

Tabela 2. Propriedades físico-químicas do cimento

\begin{tabular}{lc}
\multicolumn{1}{c}{ Propriedade } & Valor \\
\hline$\% \mathrm{MgO}$ & 4,11 \\
$\% \mathrm{SO}_{3}$ & 2,99 \\
$\% \mathrm{CaO}$ & 60,73 \\
$\% \mathrm{Al}_{2} \mathrm{O}_{3}$ & 4,38 \\
$\% \mathrm{Fe}_{2} \mathrm{O}_{3}$ & 2,83 \\
$\% \mathrm{~S}_{\mathrm{i}}$ & 19,9 \\
$\%$ Resíduo insolúvel & 0,77 \\
Resistência aos 7 dias & 42 \\
(MPa) & \\
Resistência aos 28 dias & 53 \\
(MPa) & \\
$\%$ Finura & 0,04 \\
$\mathrm{Gs}$ & 3,11 \\
\hline
\end{tabular}

\subsection{Definição dos teores de cimento, pontos de moldagem e tempo de cura}

Os pontos de moldagem foram estabelecidos após a realização dos ensaios de compactação do solo e das misturas solocimento nas três energias: normal, intermediária e modificada, de acordo com a norma brasileira NBR 7182 (ABNT 2016). A Tabela 3 mostra os resultados das curvas de compactação do solo: peso específico seco aparente máximo e teor de umidade ótimo.

Tabela 3. Resultado dos ensaios de compactação do solo e das misturas solo-cimento

\begin{tabular}{cllllll}
\hline \multirow{2}{*}{$\begin{array}{c}\text { C } \\
\text { (\%) }\end{array}$} & \multicolumn{2}{l}{$\begin{array}{l}\text { Peso específico seco } \\
\text { máximo }\left(\mathbf{k N} / \mathbf{m}^{\mathbf{3}}\right)\end{array}$} & \multicolumn{2}{l}{$\begin{array}{l}\text { Teor } \\
\text { (\%) }\end{array}$} & Ie umidade ótimo \\
\cline { 2 - 7 } & SE & IE & ME & SE & IE & ME \\
\hline 0 & 13,72 & 15,43 & 16,75 & 26,5 & 20,5 & 14,5 \\
3 & 13,85 & 15,65 & 16,85 & 26 & 18 & 15 \\
5 & 13,8 & 15,55 & 17,05 & 26,5 & 18 & 15 \\
7 & 14 & 15,55 & 16,95 & 26 & 18,5 & 14,5 \\
9 & 14 & 15,55 & 16,95 & 25,5 & 18 & 15 \\
\hline
\end{tabular}

Com o objetivo de estudar a influência da massa específica seco e dos vazios sobre a resistência mecânica do solo cimentado artificialmente com o cimento mencionado, foram definidos 3 pontos de moldagem: SE (energia normal), IE (energia intermediária) e ME (energia modificada). Estes pontos de moldagem foram definidos estrategicamente considerando possíveis condições de campo. Pontos de moldagem estratégicos para estudar solos melhorados já foram utilizados anteriormente por Rios et al. (2012), Baldovino et al. (2018c), Baldovino et al. (2018a), Baldovino et al. (2018b) e Moreira et al. (2018). Todos os corpos de prova de solo-cimento foram submetidos a ensaios depois de 7 dias de cura em condições de saturação para anular o 
máximo possível a influência da sucção sobre a resistência nas misturas.

\subsection{Preparação das amostras}

Para os ensaios de compressão simples foram moldados corpos de prova de $100 \mathrm{~mm}$ de altura e $50 \mathrm{~mm}$ de diâmetro (volume de $196.35 \mathrm{~cm}^{3}$ ). Depois da coleta em campo, o solo foi seco, totalmente, em estufa à temperatura de $100 \pm 5^{\circ} \mathrm{C}$, e colocado em porções uniformemente distribuídas para ser misturado com o cimento. Adicionou-se a quantidade de cimento seco com referência ao peso seco da amostra de solo em quatro teores diferentes de adição (3, 5, 7 e 9\%) de acordo a estudos prévios (Consoli et al., 2007; Rios et al., 2013). Realizou-se a mistura do solo com o cimento de modo que a mistura ficasse a mais homogênea possível. Em seguida, foi adicionada uma porcentagem de água em massa, sendo esta porcentagem referente ao teor de umidade dos pontos de moldagem estabelecidos na Tabela 3. A mistura do solo-cimento com a água destilada foi realizada em um período não superior a 5 minutos, com isto tentando minimizar as reações do cimento com a água antes do processo de moldagem dos corpos de prova. As amostras para a moldagem dos corpos de prova foram compactadas estaticamente em três camadas com um molde de aço inox com diâmetro interno de $50 \mathrm{~mm}$, altura de $100 \mathrm{~mm}$ e espessura de $5 \mathrm{~mm}$, nas condições de compactação mostradas na Tabela 3. Para assegurar a massa específica seco máximo, obtido durante os ensaios de compactação, foi realizado o cálculo do volume do molde e da massa da mistura úmida necessários para cada corpo de prova. Após esses cálculos, foi pesada a quantidade necessária de material para cada corpo de prova. Foram necessárias 3 camadas para compactar os corpos de prova. A moldagem foi feita com a ajuda de uma prensa hidráulica manual. Depois de cada processo de moldagem, três amostras da mistura foram utilizadas para medir o teor de umidade em estufa durante 24 horas.

Os corpos de prova foram pesados em uma balança de precisão de 0,01 $\mathrm{g}$ e as dimensões do mesmo eram medidas com o uso de um paquímetro de $0,1 \mathrm{~mm}$ de precisão. Os corpos de provas extraídos foram envolvidos com plástico filme transparente para manter o teor de umidade. Por último, os corpos de prova foram armazenados em câmara úmida para processo de cura durante 6 dias (a temperatura média de $25^{\circ} \mathrm{C}$ ) para prevenir mudanças significativas de umidade até o dia do ensaio. As amostras tinham que respeitar as seguintes tolerâncias máximos para serem usadas nos ensaios de compressão simples: dimensões das amostras com diâmetro de $\pm 0,5 \mathrm{~mm}$ e altura de $\pm 1 \mathrm{~mm}$, massa específica aparente seco $\left(\gamma_{d}\right)$ de $\pm 1 \%$ e teor de umidade $(\omega)$ de $\pm 0,5 \%$. Para cada ponto de moldagem e teor de cimento foram moldados 3 corpos de prova. Após os 6 dias de cura, os corpos de prova eram imersos em um tanque com água destilada durante 24 horas antes do ensaio para garantir a sua saturação e evitar com isto a influência da sucção na resistência. Depois da imersão, eram secados superficialmente com um pano seco. Assim, todas as amostras foram curadas durante 7 dias.

\subsection{Ensaios de resistência mecânica}

Para a realização dos ensaios de compressão simples foi usada uma prensa automática com anéis calibrados para carga axial com capacidade de $4,5 \mathrm{kN}$ e $10 \mathrm{kN}$. Os ensaios foram feitos com um sistema automatizado, medindo, principalmente, a força aplicada, com resolução de $2,5 \mathrm{~N}$, a deformação, com sensibilidade de $0,01 \mathrm{~mm}$, sendo a velocidade do ensaio de $1 \mathrm{~mm} / \mathrm{min}$. Os procedimentos dos ensaios de compressão simples seguiram a norma brasileira NBR 5739 (ABNT 2018). A resistência a compressão simples é o valor da carga máxima de ruptura do material ou o valor da pressão correspondente à carga na qual ocorre deformação específica do corpo de prova de solo de $20 \%$, naqueles casos em que a curva tensão-deformação axial não apresenta um pico máximo. Adota-se a 
resistência à compressão não confinada ou simples $\left(q_{u}\right)$ de acordo com a Equação 1, quando, no ensaio a curva tensãodeformação axial, alcança um pico máximo:

$$
q_{\mathrm{u}}=\frac{\mathrm{P}_{\mathrm{R}}}{\mathrm{A}_{\mathrm{T}}}
$$

Onde $P_{R}$ é a carga de ruptura no pico da curva tensão-deformação axial e $A_{T}$ é a área da seção transversal corrigida do corpo de prova. Para a realização dos ensaios de tração foi usada uma prensa automática com capacidade de $30 \mathrm{kN}$. Adota-se a resistência à tração $\left(q_{t}\right)$ de acordo com a Equação 2, quando, no ensaio a curva tensãodeformação axial, alcança-se um pico máximo:

$$
\mathrm{q}_{\mathrm{t}}=\frac{2 \mathrm{P}_{\mathrm{R}}}{\pi \mathrm{DH}}
$$

Onde $P_{R}$ é a carga de ruptura no pico da curva tensão-deformação diametral, $\mathrm{D}$ e $\mathrm{H}$ são o diâmetro e a altura do corpo de prova, respectivamente. Os procedimentos dos ensaios de resistência à tração $\left(q_{t}\right)$ seguiram norma brasileira NRB 7222 (ABNT 2011).

\section{APRESENTAÇÃO, ANÁLISE E DISCUSSÃO DOS RESULTADOS}

As Figuras 1(a)-1(b) mostram os resultados de $q_{u}$ e $q_{t}$ das amostras depois de 7 dias de cura com a variação dos teores de cimento CP $\vee$ de 3 até $9 \%$. Nota-se que com o aumento da massa específica seco das amostras aumenta-se a resistência à compressão simples e tração, assim como com o aumento do teor de cimento também há um aumento na resistência à compressão simples e tração indireta. $\mathrm{O}$ valor de $\mathrm{q}_{\mathrm{u}}$ máximo obtido com o cimento foi de 2000 $\mathrm{kPa}$ e para $\mathrm{q}_{\mathrm{t}}$ foi de $375 \mathrm{kPa}$. Analisa-se nas Figuras 1(c)-1(d) influência da porosidade inicial sobre $q_{u}$ e $q_{t}$ das misturas solocimento. Observa-se uma variação na porosidade de $36 \%$ a $48 \%$ para $3 \%, 5 \%$, $7 \%$ e $9 \%$ de cimento. Há, também, uma relação inversamente proporcional da porosidade com $q_{u}$ e $q_{t}$, pois à medida que a porosidade diminui, o $q_{u}$ e o $q_{t}$ aumentam.

A melhor maneira de caracterizar a variação da porosidade e os resultados da resistência à compressão simples e tração observados foi através da determinação de uma curva de regressão que pudesse representar, de forma satisfatória, os resultados. A curva de regressão que melhor representou os pontos foi uma curva linear. Em média, a redução de 8 pontos porcentuais na porosidade dos corpos de prova solo-cimento aumentou em $1050 \mathrm{kPa}$ e $123 \mathrm{kPa}$ a resistência à compressão simples e tração das amostras, respectivamente. Ingles e Metcalf (1972); Moore et al. (1970) e da Rocha et al. (2014) também reportaram em seus estudos que a diminuição da porosidade com o aumento de $q_{u}$ e $q_{t}$.

É observada nas Figuras 2(a)-2(b) a influência da relação porosidade/teor volumétrico de cimento na resistência à compressão simples e tração por compressão diametral. O teor volumétrico de cimento (Equação 3) é definido como a razão entre volume cimento pelo volume de um corpo de prova. 
Figura 1. Efeitos da adição de cimento na resistência das misturas solo-cimento (a) Influência do teor de cimento sobre $q_{u}$. (b) Influência do teor de cimento sobre $q_{t}$. (c) Influência da porosidade sobre $q_{u} \cdot(d)$ Influência da porosidade sobre $q_{t}$.

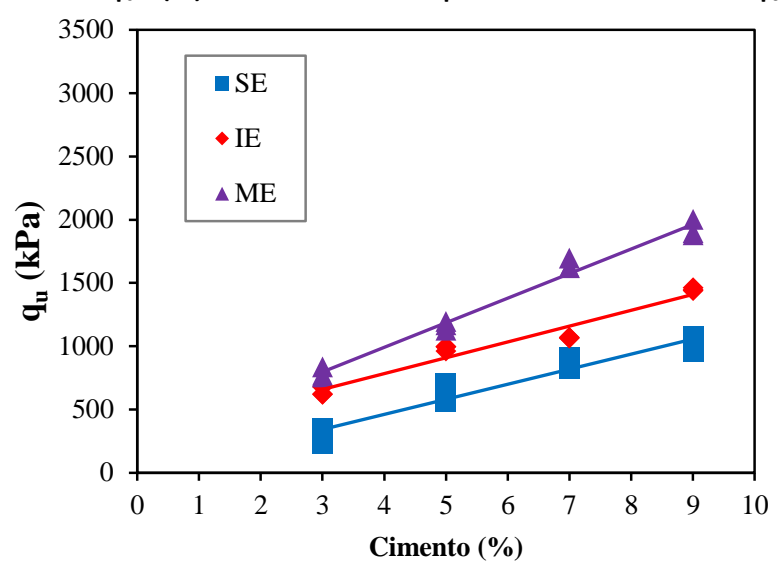

(a)

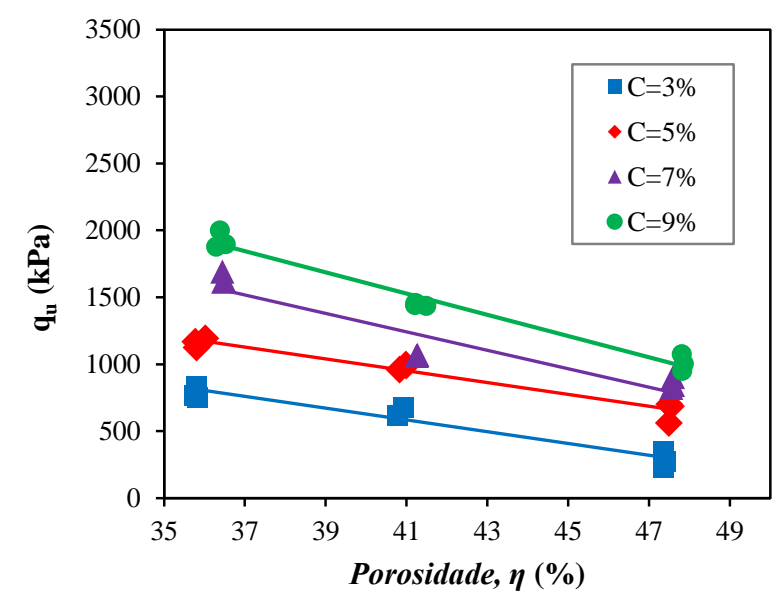

(c)

$$
C_{i v}=\left(\frac{V_{d}}{1+c / 100}\left(\frac{C}{100}\right)\right) / G_{s c}
$$

Onde $\gamma_{d}$ é o peso específico seco aparente da mistura, $\mathrm{C}$ é o teor de cimento e $\mathrm{G}_{\mathrm{sc}}$ é a densidade dos grãos do cimento. $\mathrm{O}$ teor volumétrico de aumenta com o aumento do teor de cimento, enquanto a relação porosidade/teor volumétrico diminui. Para as misturas, a relação $\eta / C_{i v}$ varia de 10,1 13,$6 ; 12,7-17,1 ; 17,5-23,5$ e de $28,5-38,2$ para 9, 7, 5 e $3 \%$ de cimento, respectivamente, tanto para $\mathrm{q}_{\mathrm{u}}$ como para $\mathrm{q}_{\mathrm{t}}$. Em média, a faixa de variação de $\eta / C_{i v}$ para cada teor de cimento é de 3,6; 4,5; 6,3 e 10,5 para 9, 7, 5 e $3 \%$, respectivamente. Ou seja, a faixa de variação cresce com a diminuição da quantidade de cimento no corpo de prova, e, o quando a faixa de variação diminui fornece os maiores valores de resistência mecânica.

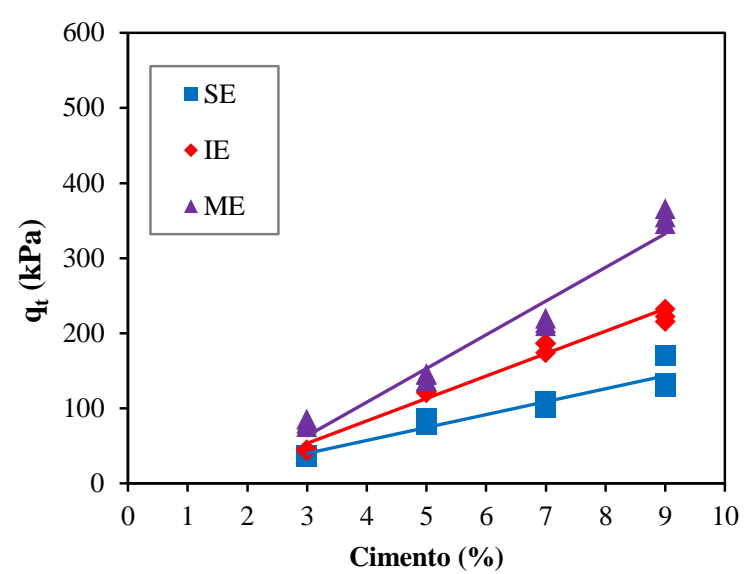

(b)

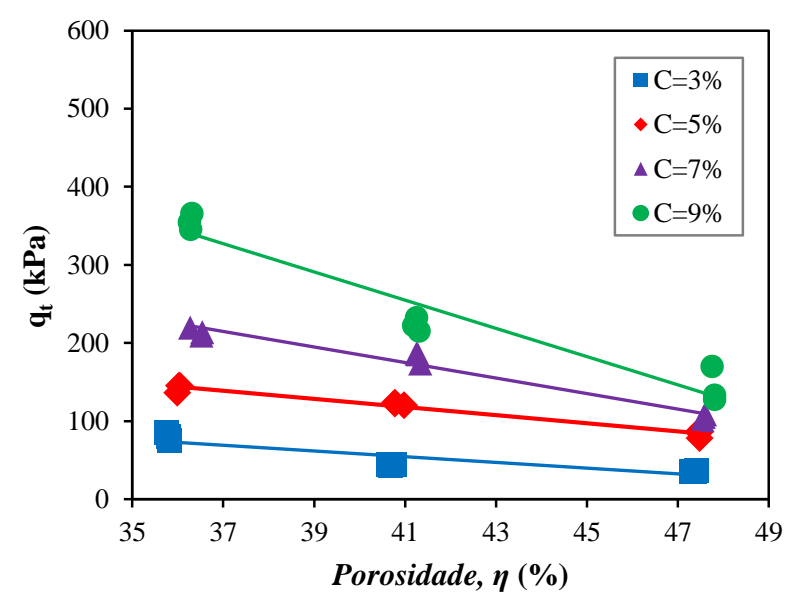

(d)

Pode-se observar nas Figuras 2(a)-2(b) que existe uma tendência potencial de $q_{u}$ e $q_{t}$ dependente de $\eta / C_{i v}$ para todos os teores de cimento. A Figura 2 mostra que comparando a redução de 20 pontos porcentuais de $\eta / C_{i v}$ se obtém que, por exemplo, se se reduz a relação vazios/cimento de uma mistura de $\eta / C_{i v}=35$ a $\eta / C_{i v}=15$ aumenta a sua resistência $q_{u}$ em $1230 \mathrm{kPa}$ e sua resistência $q_{t}$ em 210 $\mathrm{kPa}$.

Consoli et al. (2013) sugere que uma melhor tendência dos pontos experimentais de resistência à compressão simples de misturas solo-cimento pode ser obtida se o valor de $C_{i v}$ é ajustado a um exponente entre 0,01 e 1,00 dentro da faixa da relação $\eta / C_{i v}$ específica estudada. Desta maneira, os valores reportados na Figura 2 foram ajustados a um exponente decimal entre 0,01 
e 1,00 sendo o exponente de 0,45 o qual forneceu 0 melhor coeficiente de determinação para o cimento empregado. Assim, os valores de $q_{u}$ e $q_{t}$ dependendo da relação $\eta / C_{i v}{ }^{0,45}$ para os cimentos são apresentados na Figura 2.
As Equações 4 e 5 definem o crescimento de $q_{u}$ e $q_{t}$ com a diminuição de $\eta / C_{i v}{ }^{0,45}$, respectivamente:

Figura 2. Efeitos da relação porosidade/teor volumétrico de cimento sobre a resistência mecânica das misturas solo-cimento: (a) sobre a compressão simples (b) sobre a tração indireta

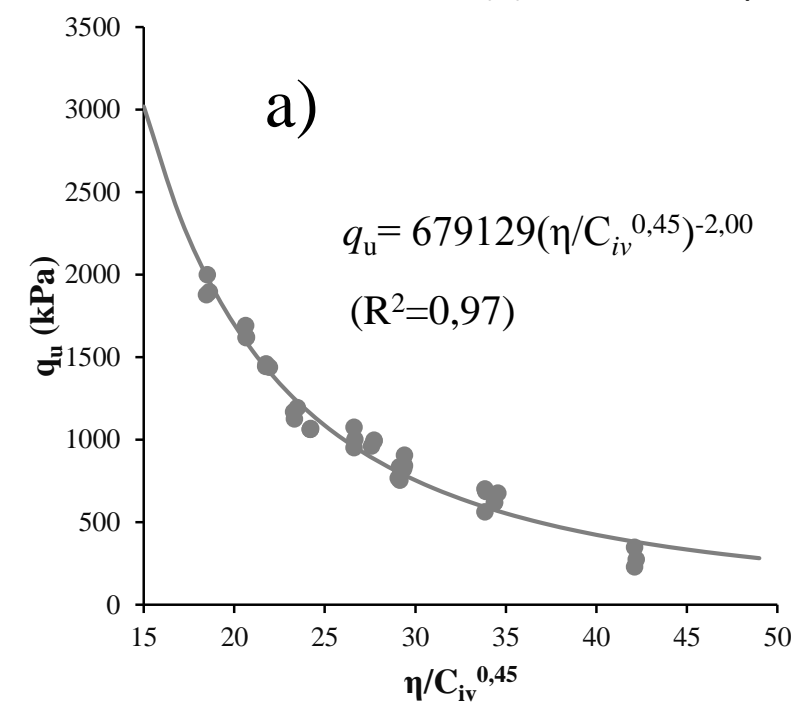

$$
q_{u}=679129\left[\frac{\eta}{\left(C_{i v}\right)^{0,45}}\right]^{-2,00}\left(R^{2}=0,97\right)
$$$$
q_{t}=99757\left[\frac{\eta}{\left(C_{i v}\right)^{0,45}}\right]^{-2,00}\left(R^{2}=0,88\right)
$$

Pode-se observar que as Equações $4 \mathrm{e}$ 5 seguem a forma: $q_{u}=A\left[\frac{\eta}{C_{i v}{ }^{C}}\right]^{-B}$. Baseado nos estudos feitos por Consoli et al. (2013) e Baldovino (2018d), os valores de B e C $\frac{q_{\mathrm{u}}}{q_{\mathrm{u}}\left(\frac{\eta}{c_{\mathrm{iv}}{ }^{\mathrm{C}}}=\Omega\right)}$ ou $\frac{q_{\mathrm{t}}}{q_{\mathrm{t}}\left(\frac{\eta}{C_{\mathrm{iv}}{ }^{\mathrm{C}}}=\Omega\right)}$ $=\frac{A\left(\eta / C_{i v}{ }^{C}\right)^{-B}}{A(\Omega)^{-B}}=(\Omega)^{B}\left(\eta / C_{i v}{ }^{C}\right)^{-B}$

depende do tipo de solo. Consegue-se obter um mesmo comportamento dos resultados de compressão simples e tração com o uso da relação $\eta / C_{i v}{ }^{0,45}$. A relação $\eta / C_{i v}{ }^{C}$ é capaz de fornecer uma tendência única da resistência do solo siltoso experimentado no presente trabalho cimentado artificialmente com um cimento de alta resistência inicial. Para Rios et al. (2012) e Mola-Abasi e Shooshpasha (2016) a relação $\eta / C_{i v}$

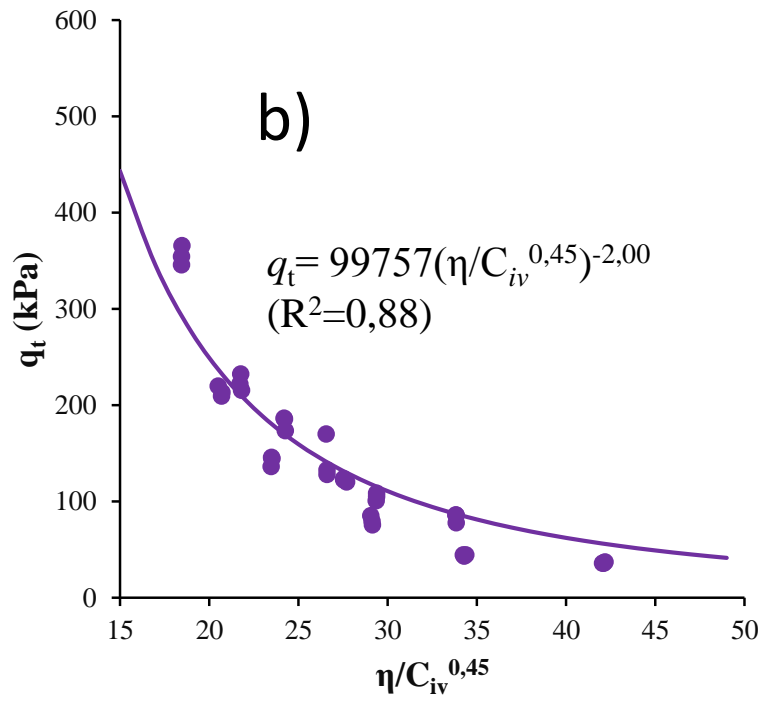

demonstra ser um ótimo parâmetro de ajuste para descrever o comportamento à compressão não confinada de solos estabilizados com cimento.

Outra forma de representar um crescimento único da resistência $q_{u}$ e $q_{t}$ dos três tipos de solos é mediante a normalização (divisão) das resistências. Segundo Consoli et al. (2013) a normalização é obtida dividindo a Equação 4 ou 5 por um valor específico arbitrário de resistência à compressão simples e à tração, correspondente a um valor de um dado ajustado de porosidade, $\eta / C_{\mathrm{iv}}{ }^{\mathrm{C}}=\Omega$, que leva à Equação 6:

O valor de $\Omega$ pode ser escolhido na faixa de variação de $\eta / C_{\text {iv }}{ }^{C}$ média para tração e para compressão de acordo à Figura 2 (isto é, entre 18-42). Para o presente trabalho foi escolhido o 27,5. Assim, com o valor de $\Omega=$ 27,5, a Equação 6 se converte na Equação 7: 
$\frac{q_{u}}{q_{u}\left(\frac{\eta}{c_{i v}{ }^{C}}=27,5\right)}$ ou $\frac{q_{t}}{q_{t}\left(\frac{\eta}{C_{i v}{ }^{C}}=27,5\right)}=A\left(n / C_{i v}{ }^{C}\right)^{-B}$

Cada valor real de $q_{t}$ e $q_{u}$ deve ser divido pela sua respectiva resistência de normalização resultante de valor específico de $\eta / C_{\text {iv }}{ }^{C}=27,5$ e calculado com as expressões que seguem a forma da Equação 4 e 5 . Quando cada valor experimental de resistência é dividido pela sua respectiva resistência de normalização é obtido um valor quociente. Os valores quocientes de compressão e tração indireta das misturas estudadas formam uma mesma tendência potencial descrita pela Equação 8 e mostrada na Figura 3 junto com a normalização das resistências. Assim, a Equação 7 converte-se e, uma equação única (Equação 8) que descreve o comportamento de $\mathrm{q}_{\mathrm{t}}$ e $\mathrm{q}_{\mathrm{u}}$ :

$\frac{q_{u}}{\left(\frac{\eta}{c_{\text {iv }}{ }^{c}}=27,5\right)}$ ou $\frac{q_{t}}{q_{t}\left(\frac{\eta}{c_{i v}{ }^{C}}=27,5\right)}=756,25\left(\eta / c_{\text {iv }}{ }^{0,45}\right)^{-2,00}$

Figura 3. Normalização da compressão simples $\left(q_{u}\right)$ e da tração indireta $\left(q_{t}\right)$ [para toda a gama de $\eta / \mathcal{C}_{i v}{ }^{0.45}$ ] dividindo por $\mathrm{q}_{\mathrm{u}}$ e $\mathrm{q}_{\mathrm{t}}$ em $\eta / \mathrm{C}_{i v}{ }^{0,45}=35$ considerando todas as misturas solo-cimento usando 7 dias de cura e diferentes energias de compactação.

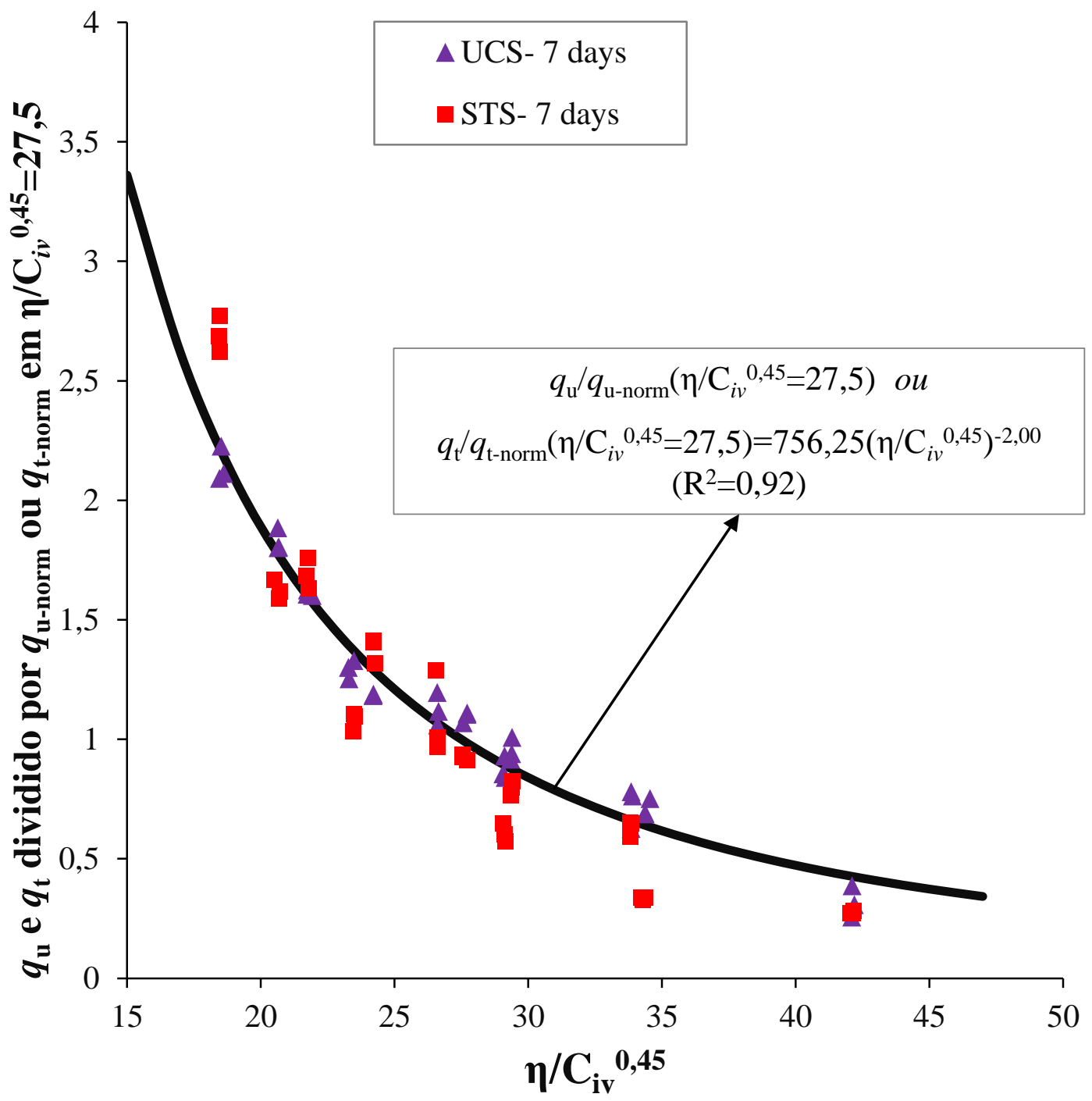

Uma relação empírica entre a compressão e a tração pode ser calculada em termos das Equações 4-5 das amostras solocimento. Esta relação pode ser chamada de 
$\xi=q_{t} / q_{u}$, e é independente da relação $\eta /\left(C_{i v}\right)^{0,45}$. Desta maneira, as Equações que descrevem o crescimento de $q_{u}$ e $q_{t}$ em função de $\eta /\left(C_{i v}\right)^{0,45}$ (Ver Figuras 2a-2b) podem ser expressas como uma relação direta de $q_{t} / q_{u}$ para o solo utilizado. A relação $\mathrm{q}_{\mathrm{t}} / \mathrm{q}_{\mathrm{u}}$ assegura uma constante decimal, a qual é calculada na Equação 9. De forma geral, $\xi$ obtém um valor de 0,15. Assim, $\mathrm{q}_{\mathrm{t}}$ demonstra ser uma porcentagem de $15 \%$ do valor de $q_{u}$ para o silte amarelo estudado.

$$
\frac{q_{t}}{q_{u}}=\frac{99757\left[\frac{\eta}{\left(C_{i v}\right)^{0,45}}\right]^{-2,00}}{679129\left[\frac{\eta}{\left(C_{i v}\right)^{0,45}}\right]^{-2,00}}=0,15
$$

\section{CONSIDERAÇÕES FINAIS}

De acordo com o tipo de solo usado na presente pesquisa (siltoso), o tipo de cimento, os teores de cimento (3-9\%) e o tempo de cura de 7 dias a que foram submetidos os corpos de prova, além das análises dos resultados, as seguintes conclusões são abordadas:

i). A resistência à compressão simples dos corpos de prova de misturas solocimento aumentou com o acréscimo do teor de cimento e com o aumento da massa específica seco de moldagem. Além disso, uma tendência linear foi a melhor forma de representar o crescimento de $q_{\mathrm{u}}$ com a variação do teor de cimento de 3 a $9 \%$. Por outra parte, a diminuição da porosidade das amostras também fez aumentar $q_{\mathrm{u}}$.

ii). A relação porosidade/teor volumétrico de cimento $\left(n / C_{i v}\right)$ demonstrou ser um parâmetro eficiente para estudar o comportamento mecânico das misturas como foi reportado em estudos prévios. Um exponente de 0,45 sobre o teor volumétrico de cimento $\left(n / C_{i v}{ }^{0,45}\right)$ forneceu melhor ajuste das amostras ensaiadas à compressão simples. Além disso uma relação tração/compressão de 0,15 foi calculada.

\section{AGRADECIMENTOS}

Os autores gostariam de agradecer o apoio da CAPES, CNPq e Fundação Araucária do Paraná.

\section{REFERÊNCIAS}

ASTM. ASTM D 2487 - 11 Standard Practice for Classification of Soils for Engineering Purposes (Unified Soil Classification System). 2011, ASTM International, West Conshohocken, Pa, D5521-5, 1-5.

ASTM. ASTM D 854 - 14 Standard Test Methods for Specific Gravity of Soil Solids by Water Pycnometer 1. 2014, ASTM International, West Conshohocken, $\mathrm{Pa}$.

BALDOVINO, J. A. Comportamento mecânico de um solo siltoso da Formação geológica Guabirotuba tratado com cal em diferentes tempos de cura. 2018d. Dissertação (Mestrado) Universidade Tecnológica Federal do Paraná, Curitiba/PR. Disponível em: < http://repositorio.utfpr.edu.br/jspui/handle/1/34 08>.Acesso: 26 janeiro 2019.

BALDOVINO, J. A., MOREIRA, E. B., e IZZO, R. L. S. Discussion of 'Control factors for the long term compressive strength of lime treated sandy clay soil' . Transportation Geotechnics, 15, 1-3, 2018b.

https://doi.org/10.1016/j.trgeo.2017.11.002

BALDOVINO, J. A., MOREIRA, E. B., IZZO, R. L. DOS S., e ROSE, J. L. Empirical Relationships with Unconfined Compressive Strength and Split Tensile Strength for the Long Term of a LimeTreated Silty Soil. Journal of Materials in Civil Engineering, 30(8), 6018008, 2018a. https://doi.org/10.1061/(ASCE)MT.1943$\underline{5533.0002378}$

BALDOVINO, J. A., MOREIRA, E. B., TEIXEIRA, W., IZZO, R. L. S., e ROSE, J. L. Effects of lime addition on geotechnical properties of sedimentary soil in Curitiba, Brazil. Journal of Rock Mechanics and Geotechnical Engineering, 10(1), 188-194, 2018c.

https://doi.org/10.1016/j.jrmge.2017.10.001

ASOCIAÇÃO BRASILEIRA DE NORMAS TÉCNICAS 
(ABNT). Solo-Determinação do limite de liquidez. NBR 6459: 2016. Rio de Janeiro, Brasil.

ASOCIAÇÃO BRASILEIRA DE NORMAS TÉCNICAS (ABNT). Solo - Determinação do limite de plasticidade. NBR 7180: 2016, Rio de Janeiro, Brasil.

ASOCIAÇÃO BRASILEIRA DE NORMAS TÉCNICAS (ABNT). Cimento Portland e outros materiais em pó - Determinação da massa específica. NBR 16605: 2017, Rio de Janeiro, Brasil.

ASOCIAÇÃO BRASILEIRA DE NORMAS TÉCNICAS (ABNT). Solo - Ensaio de compactação. NBR 7182: 2016, Rio de Janeiro, Brasil.

ASOCIAÇÃO BRASILEIRA DE NORMAS TÉCNICAS (ABNT). Concreto e argamassa - Determinação da resistência à tração por compressão diametral de corpos de prova cilíndricos. NBR 7222: 2011, Rio de Janeiro, Brasil.

ASOCIAÇÃO BRASILEIRA DE NORMAS TÉCNICAS (ABNT). (2007). Concreto - Ensaio de compressão de corpos de prova cilíndricos. NBR 5739: 2018, Rio de Janeiro, Brasil.

CONSOLI, N. C., FESTUGATO, L., DA ROCHA, C. G., e CRUZ, R. C. Key parameters for strength control of rammed sand-cement mixtures: Influence of types of portland cement. Construction and Building Materials, 49, 591-597, 2013. https://doi.org/10.1016/j.conbuildmat.2013.08.0 $\underline{62}$

CONSOLI, N. C., FOPPA, D., FESTUGATO, L., e HEINECK, K. S. Key Parameters for Strength Control of Artificially Cemented Soils. Journal of Geotechnical and Geoenvironmental Engineering, 133(2), 197-205, 2007. https://doi.org/10.1061/(ASCE)10900241(2007)133:2(197)

DA ROCHA, C. G., CONSOLI, N. C., e DALLA ROSA JOHANN, A. Greening stabilized rammed earth: devising more sustainable dosages based on strength controlling equations. Journal of Cleaner Production, 66, 19-26. https://doi.org/10.1016/j.jclepro.2013.11.041

FIROOZI, A. A., GUNEY OLGUN, C., FIROOZI, A. A., e BAGHINI, M. S. Fundamentals of soil stabilization. International Journal of Geo-
Engineering, 8(1), 26, 2017. https://doi.org/10.1186/s40703-017-0064-9

HORPIBULSUK, S., RACHAN, R., CHINKULKIJNIWAT, A., RAKSACHON, Y., e SUDDEEPONG, A. Analysis of strength development in cement-stabilized silty clay from microstructural considerations. Construction and Building Materials, 24(10), 2011-2021, 2010. https://doi.org/10.1016/j.conbuildmat.2010.03.0 $\underline{11}$

INGLES, O. G., e METCALF, J. B. Soil stabilization-Principles and practice. Butterworths, Melbourne, Australia, 1972.

JIN, L., SONG, W., SHU, X., e HUANG, B. Use of water reducer to enhance the mechanical and durability properties of cement-treated soil. Construction and Building Materials, 159, 690694,

2018.

https://doi.org/10.1016/j.conbuildmat.2017.10.1 $\underline{20}$

LADE, P. V., e TRADS, N. The role of cementation in the behaviour of cemented soils. Geotechnical Research, 1(4), 111-132, 2014. https://doi.org/10.1680/gr.14.00011

MOLA-ABASI, H., e SHOOSHPASHA, I. Influence of zeolite and cement additions on mechanical behavior of sandy soil. Journal of Rock Mechanics and Geotechnical Engineering, 8(5), 746-752 2016.

https://doi.org/10.1016/j.jrmge.2016.01.008

MOORE, R. K., KENNEDY, T. W. AND HUDSON, W. $R$. Factors affecting the tensile strength of cement-treated materials. Highway Research Record: Soil Stabilization: Multiple Aspects, Vol. 315, Highway Research Board, Washington, D.C., 64-80, 1970.

MOREIRA, E. B., BALDOVINO, J. A., ROSE, J. L., e LUIS DOS SANTOS IZZO, R. Effects of porosity, dry unit weight, cement content and void/cement ratio on unconfined compressive strength of roof tile waste-silty soil mixtures. Journal of Rock Mechanics and Geotechnical Engineering, 11(2), 369-378, 2018. https://doi.org/10.1016/i.jrmge.2018.04.015

PAKBAZ, M. S., e ALIPOUR, R. Influence of cement addition on the geotechnical properties of an Iranian clay. Applied Clay Science, 67-68, 1-4, 
2012. https://doi.org/10.1016/i.clay.2012.07.006

POR, S., NISHIMURA, S., e LIKITLERSUANG, S. Deformation characteristics and stress responses of cement-treated expansive clay under confined one-dimensional swelling. Applied Clay Science, 146, 316-324, 2017.

https://doi.org/10.1016/j.clay.2017.06.022

RIOS, S., VIANA DA FONSECA, A., e BAUDET, B. A. Effect of the Porosity/Cement Ratio on the Compression of Cemented Soil. Journal of Geotechnical and Geoenvironmental Engineering, 138(11), 1422-1426, 2012. https://doi.org/10.1061/(ASCE)GT.1943$\underline{5606.0000698}$

RIOS, S., VIANA DA FONSECA, A., CONSOLI, N. C., FLOSS, M., e CRISTELO, N. Influence of grain size and mineralogy on the porosity/cement ratio. Géotechnique Letters, 3(3), 130-136, 2013. https://doi.org/10.1680/geolett.13.00003

RONOH, V., TOO, J. K., KALULI, J. W., e VICTOR, $M$. R. Cement effects on the physical properties of expansive clay soil and the compressive strength of compressed interlocking clay blocks. Eur Int J Sci Technol, 3(8), 74-82, 2014. 